\title{
A fine quantum mechanism of classical gravity
}

\author{
By Michael A. Ivanov \\ Chair of Physics, \\ Belarus State University of Informatics and Radioelectronics, \\ 6 P. Brovka Street, BY 220027, Minsk, Republic of Belarus. \\ E-mail: ivanovma@gw.bsuir.unibel.by.
}

November 17, 2018

\begin{abstract}
It is shown that screening the background of super-strong interacting gravitons ensures the Newtonian attraction, if a part of single gravitons is pairing and graviton pairs are destructed by collisions with a body. If the considered quantum mechanism of classical gravity is realized in the nature, than an existence of black holes contradicts to the equivalence principle. In such the model, Newton's constant is proportional to $H^{2} / T^{4}$, where $H$ is the Hubble constant, $T$ is an equivalent temperature of the graviton background. The estimate of the Hubble constant is obtained for the Newtonian limit: $H=3.026 \cdot 10^{-18} \mathrm{~s}^{-1}$ (or $94.576 \mathrm{~km} \cdot \mathrm{s}^{-1} \cdot M p c^{-1}$ ).
\end{abstract}

PACS 04.60.-m, 98.70.Vc

\section{Introduction}

It was shown by the author in the previous study [1, 2] that an alternative explanation of cosmological redshift as a result of interaction of a photon with the graviton background is possible. In the case, observed dimming of supernovae Ia [3] and the Pioneer 10 anomaly [4] may be explained from one point of view as additional manifestations of interaction with the graviton 
background. Some primary features of a new cosmological model, based on this approach, are described in author's preprint [5].

In this study (for details, see author's full paper [6]), forces of gravitonic radiation pressure are considered which act on bodies in a presence of such the background. It is shown that pressure of single gravitons of the background, which run against a body pair from infinity, results in mutual attraction of bodies with a magnitude which should be approximately 1000 times greater than Newtonian attraction. But pressure of gravitons scattered by bodies gives a repulsion force of the same order; the last is almost exact compensating this attraction. To get Newton's law of gravity, it is necessary to assume that gravitons form correlated pairs. By collision with a body, such a pair should destruct in single gravitons. Flying away gravitons of a pair should happen in independent directions, that decreases a full cross-section of interaction with scattered gravitons. As a result, an attraction force will exceed a corresponding repulsion force acting between bodies. In such the model, Newton's constant is connected with the Hubble constant that gives a possibility to obtain a theoretical estimate of the last. We deal here with a flat non-expanding universe fulfilled with superstrong interacting gravitons; it changes the meaning of the Hubble constant which describes magnitude of three small effects of quantum gravity but not any expansion.

\section{Screening the graviton background}

If gravitons of the background run against a pair of bodies with masses $m_{1}$ and $m_{2}$ (and energies $E_{1}$ and $E_{2}$ ) from infinity, then a part of gravitons is screened. Let $\sigma\left(E_{1}, \epsilon\right)$ is a cross-section of interaction of body 1 with a graviton with an energy $\epsilon=\hbar \omega$, where $\omega$ is a graviton frequency, $\sigma\left(E_{2}, \epsilon\right)$ is the same cross-section for body 2 . In absence of body 2 , a whole modulus of a gravitonic pressure force acting on body 1 would be equal to:

$$
4 \sigma\left(E_{1},<\epsilon>\right) \cdot \frac{1}{3} \cdot \frac{4 f(\omega, T)}{c},
$$

where $f(\omega, T)$ is a graviton spectrum with a temperature $T$ (assuming to be planckian), the factor 4 in front of $\sigma\left(E_{1},<\epsilon>\right)$ is introduced to allow all possible directions of graviton running, $\langle\epsilon\rangle$ is another average energy of running gravitons with a frequency $\omega$ taking into account a probability of that in a realization of flat wave a number of gravitons may be equal to zero, and that not all of gravitons ride at a body. 
Body 2, placed on a distance $r$ from body 1, will screen a portion of running against body 1 gravitons which is equal for big distances between the bodies (i.e. by $\left.\sigma\left(E_{2},<\epsilon>\right) \ll 4 \pi r^{2}\right)$ :

$$
\frac{\sigma\left(E_{2},<\epsilon>\right)}{4 \pi r^{2}}
$$

Taking into account all frequencies $\omega$, an attractive force will act between bodies 1 and 2 :

$$
F_{1}=\int_{0}^{\infty} \frac{\sigma\left(E_{2},<\epsilon>\right)}{4 \pi r^{2}} \cdot 4 \sigma\left(E_{1},<\epsilon>\right) \cdot \frac{1}{3} \cdot \frac{4 f(\omega, T)}{c} d \omega
$$

Let $f(\omega, T)$ is described with the Planck formula, $x \equiv \hbar \omega / k T$, and $\bar{n} \equiv$ $1 /(\exp (x)-1)$ is an average number of gravitons in a flat wave with a frequency $\omega$ (on one mode of two distinguishing with a projection of particle spin). Let $P(n, x)$ is a probability of that in a realization of flat wave a number of gravitons is equal to $n$, for example $P(0, x)=\exp (-\bar{n})$.

If $P_{1}$ is a probability that a single graviton will ride namely at the body and one assume that $P_{1}=P(1, x)$, where $P(1, x)=\bar{n} \exp (-\bar{n})$, (below it is admitted for pairing gravitons: $P_{1}=P(1,2 x)$ - see section 4$)$, then the quantity $\left\langle\epsilon>\right.$ is equal to: $\langle\epsilon\rangle=\hbar \omega(1-P(0, x)) \bar{n}^{2} \exp (-\bar{n})$. Then we get for an attraction force $F_{1}: F_{1} \equiv G_{1} \cdot m_{1} m_{2} / r^{2}$ where the constant $G_{1}$ is equal by $T=2.7 \mathrm{~K}$ to $[6] G_{1}=1215.4 \cdot G$, that is three order greater than Newton's constant $G$.

But if gravitons are elastic scattered with body 1, then our reasoning may be reversed: the same portion (2) of scattered gravitons will create a repulsive force $F_{1}^{\prime}$ acting on body 2 and equal to $F_{1}^{\prime}=F_{1}$, if one neglects with small allowances which are proportional to $D^{3} / r^{4}$ (see section 5 ).

So, for bodies which elastic scatter gravitons, screening a flux of single gravitons does not ensure Newtonian attraction. But for black holes which absorb any particles and do not re-emit them, we will have $F_{1}^{\prime}=0$. It means that such the object would attract other bodies with a force which is proportional to $G_{1}$ but not to $G$, i.e. Einstein's equivalence principle would be violated for it. This conclusion, as we shall see below, stays in force for the case of graviton pairing too. 


\section{Graviton pairing}

To ensure an attractive force which is not equal to a repulsive one, particle correlations should differ for in and out flux. For example, single gravitons of running flux may associate in pairs. If such pairs are destructed by collision with a body, then quantities $\langle\epsilon\rangle$ will distinguish for running and scattered particles. Graviton pairing may be caused with graviton's own gravitational attraction or gravitonic spin-spin interaction.

To find an average number of pairs $\bar{n}_{2}$ in a wave with a frequency $\omega$ for the state of thermodynamic equilibrium, one may replace $\hbar \rightarrow 2 \hbar$ by deducing the Planck formula. Then an average number of pairs will be equal to: $\bar{n}_{2}=1 /(\exp (2 x)-1)$, and an energy of one pair will be equal to $2 \hbar \omega$. It is important that graviton pairing does not change a number of stationary waves, so as pairs nucleate from existing gravitons. The question arises: how many different modes, i.e. spin projections, may have graviton pairs? It follows from the energy conservation law that composite gravitons should be distributed only in two modes $[6$. The spectrum of composite gravitons is proportional to the Planck one; an equivalent temperature of this sub-system is $T_{2} \equiv(1 / 8)^{1 / 4} T=0.5946 T$. It is important that the graviton pairing effect does not changes computed values of the Hubble constant and of anomalous deceleration of massive bodies [1]: twice decreasing of a sub-system particle number due to the pairing effect is compensated with twice increasing the cross-section of interaction of a photon or any body with such the composite gravitons.

\section{Computation of Newton's constant}

If running graviton pairs ensure for two bodies an attractive force $F_{2}$, then a repulsive force due to re-emission of gravitons of a pair alone will be equal to $F_{2}^{\prime}=F_{2} / 2$. It follows from that the cross-section $\sigma\left(E_{2},<\epsilon>\right)=\frac{1}{2} \cdot \sigma\left(E_{2},<\right.$ $\left.\epsilon_{2}\right\rangle$ ), where $\left\langle\epsilon_{2}\right\rangle$ is an average pair energy with taking into account a probability of that in a realization of flat wave a number of graviton pairs may be equal to zero, and that not all of graviton pairs ride at a body $\left(<\epsilon_{2}>\right.$ is an analog of $\langle\epsilon>$ ). We get for graviton pairs:

$$
<\epsilon_{2}>=2 \hbar \omega(1-P(0,2 x)) \bar{n}_{2}^{2} \exp \left(-\bar{n}_{2}\right) \cdot P(0, x) .
$$


Then a force of attraction of two bodies due to pressure of graviton pairs $F_{2}$ will be equal to:

$$
F_{2}=\frac{4}{3} \cdot \frac{D^{2} c(k T)^{6} m_{1} m_{2}}{\pi^{3} \hbar^{3} r^{2}} \cdot I_{2}
$$

where $I_{2}=2.3184 \cdot 10^{-6}$. The difference $F$ between attractive and repulsive forces will be equal to:

$$
F \equiv F_{2}-F_{2}^{\prime}=\frac{1}{2} F_{2} \equiv G_{2} \frac{m_{1} m_{2}}{r^{2}},
$$

where the constant $G_{2}$ is:

$$
G_{2} \equiv \frac{2}{3} \cdot \frac{D^{2} c(k T)^{6}}{\pi^{3} \hbar^{3}} \cdot I_{2} .
$$

As $G_{1}$ as well $G_{2}$ are proportional to $T^{6}$ (and $H \sim T^{5}$ ).

If one assumes that $G_{2}=G$, then by $T=2.7 K$ the constant $D$ should have the value: $D=1.124 \cdot 10^{-27} \mathrm{~m}^{2} / \mathrm{eV}^{2}$. We can establish a connection between the two fundamental constants $G$ and $H$ under the condition that $G_{2}=G$ :

$$
H=\left(G \frac{45}{32 \pi^{5}} \frac{\sigma T^{4} I_{4}^{2}}{c^{3} I_{2}}\right)^{1 / 2}=3.026 \cdot 10^{-18} s^{-1},
$$

or in the units which are more familiar for many of us: $H=94.576 \mathrm{~km} \cdot \mathrm{s}^{-1}$. $M p c^{-1}$.

This value of $H$ is significantly larger than we see in the majority of present astrophysical estimations [3, 7, but it is well consistent with some of them [8] and with the observed value of anomalous acceleration of Pioneer 10 [4] $w=(8.4 \pm 1.33) \cdot 10^{-10} \mathrm{~m} / \mathrm{s}^{2}$. Any massive body, moving relative to the background, must feel a deceleration $w \simeq H c\left[1\right.$, 2]; with $H=3.026 \cdot 10^{-18} s^{-1}$ we have $H c=9.078 \cdot 10^{-10} \mathrm{~m} / \mathrm{s}^{2}$.

\section{Why and when gravity is geometry}

The described quantum mechanism of classical gravity gives Newton's law with the constant $G_{2}$ value (7) and the connection (8) for the constants $G_{2}$ and $H$. We have obtained the rational value of $H$ by $G_{2}=G$, if the condition of big distances is fulfilled:

$$
\sigma\left(E_{2},<\epsilon>\right) \ll 4 \pi r^{2}
$$


Because it is known from experience that for big bodies of the solar system, Newton's law is a very good approximation, one would expect that the condition (9) is fulfilled, for example, for the pair Sun-Earth. But assuming $r=1 \mathrm{AU}$ and $E_{2}=m_{\odot} c^{2}$, we obtain assuming for rough estimation $<\epsilon>\rightarrow \bar{\epsilon}: \sigma\left(E_{2},<\epsilon>\right) / 4 \pi r^{2} \sim 4 \cdot 10^{12}$. It means that in the case of interaction of gravitons or graviton pairs with the Sun in the aggregate, the considered quantum mechanism of classical gravity could not lead to Newton's law as a good approximation. This "contradiction" with experience is eliminated if one assumes that gravitons interact with "small particles" of matter - for example, with atoms. If the Sun contains of $N$ atoms, then $\sigma\left(E_{2},<\epsilon>\right)=N \sigma\left(E_{a},<\epsilon>\right)$, where $E_{a}$ is an average energy of one atom. For rough estimation we assume here that $E_{a}=E_{p}$, where $E_{p}$ is a proton rest energy; then it is $N \sim 10^{57}$, i.e. $\sigma\left(E_{a},<\epsilon>\right) / 4 \pi r^{2} \sim 10^{-45} \ll 1$.

This necessity of "atomic structure" of matter for working the described quantum mechanism is natural relative to usual bodies. But would one expect that black holes have a similar structure? If any radiation cannot be emitted with a black hole, a black hole should interact with gravitons as an aggregated object, i.e. the condition (9) for a black hole of sun mass has not been fulfilled even at distances $\sim 10^{6} \mathrm{AU}$.

For bodies without an atomic structure, the allowances, which are proportional to $D^{3} / r^{4}$ and are caused by decreasing a gravitonic flux due to the screening effect, will have a factor $m_{1}^{2} m_{2}$ or $m_{1} m_{2}^{2}$. These allowances break the equivalence principle for such the bodies.

For bodies with an atomic structure, a force of interaction is added up from small forces of interaction of their "atoms":

$$
F \sim N_{1} N_{2} m_{a}^{2} / r^{2}=m_{1} m_{2} / r^{2}
$$

where $N_{1}$ and $N_{2}$ are numbers of atoms for bodies 1 and 2. The allowances to full forces due to the screening effect will be proportional to the quantity: $N_{1} N_{2} m_{a}^{3} / r^{4}$, which can be expressed via the full masses of bodies as $m_{1}^{2} m_{2} / r^{4} N_{1}$ or $m_{1} m_{2}^{2} / r^{4} N_{2}$. By big numbers $N_{1}$ and $N_{2}$ the allowances will be small. The allowance to the force $F$, acting on body 2 , will be equal to:

$$
\Delta F=\frac{1}{3 N_{2}} \cdot \frac{D^{3} c^{3}(k T)^{7} m_{1} m_{2}^{2}}{\pi^{4} \hbar^{3} r^{4}} \cdot I_{3},
$$

(for body 1 we shall have the similar expression if replace $N_{2} \rightarrow N_{1}, m_{1} m_{2}^{2} \rightarrow$ $m_{1}^{2} m_{2}$ ), where $I_{3}=1.0988 \cdot 10^{-7}$. 
Let us find the ratio:

$$
\frac{\Delta F}{F}=\frac{D E_{2} k T}{N_{2} 2 \pi r^{2}} \cdot \frac{I_{3}}{I_{2}} .
$$

Using this formula, we can find by $E_{2}=E_{\odot}, r=1 A U: \Delta F / F \sim 10^{-46}$.

An analogical allowance to the force $F_{1}$ has the order $\sim 10^{-48} F_{1}$, or $\sim 10^{-45} \mathrm{~F}$. We see that for bodies with an atomic structure the considered mechanism leads to very small deviations from Einstein's equivalence principle, if the condition (9) is fulfilled for microparticles, which prompt interact with gravitons.

For small distances we shall have:

$$
\sigma\left(E_{2},<\epsilon>\right) \sim 4 \pi r^{2} .
$$

It takes place by $E_{a}=E_{p},\left\langle\epsilon>\sim 10^{-3} \mathrm{eV}\right.$ for $r \sim 10^{-11} \mathrm{~m}$. This quantity is many order larger than the Planck length. The equivalence principle should be broken at such distances.

\section{Conclusion}

It is known that giant intellectual efforts to construct a quantum theory of metric field, based on the theory of general relativity, have not a hit until today (see [9]). From a point of view of the considered approach, one may explain it by the fact that gravity is not geometry at short distances $\sim 10^{-11} \mathrm{~m}$. Actually, it means that at such the distances quantum gravity cannot be described alone.

It follows from the present study that the geometrical description of gravity should be a good idealization at big distances by the condition of "atomic structure" of matter. This condition cannot be accepted only for black holes which must interact with gravitons as aggregated objects. In addition, the equivalence principle is roughly broken for black holes, if the described quantum mechanism of classical gravity is realized in the nature.

Other important features of this mechanism are the following ones.

- Attracting bodies are not initial sources of gravitons. In this sense, a future theory must be non-local to describe gravitons running from infinity.

- Newton's law takes place if gravitons are pairing; to get preponderance of attraction under repulsion, graviton pairs should be destructed by interaction with matter particles. 
- The described quantum mechanism of classical gravity is obviously asymmetric relative to the time inversion. By the time inversion, single gravitons would run against bodies forming pairs. It would lead to replacing a body attraction with a repulsion. But such the change will do impossible graviton pairing.

- The two fundamental constants - Newton's and Hubble's ones - are connected with each other in such the model. The estimate of Hubble's constant has been got here using an additional postulate $P_{1}=P(1,2 x)$ for pairing gravitons.

- It is proven that graviton pairs should be distributed in two modes with different spin projections.

A future theory dealing with gravitons as usual particles having an energy, a momentum etc should have a number of features, which are not characterizing any existing model, to image the recounted above features of a possible quantum mechanism of gravity.

\section{References}

[1] M.A.Ivanov, General Relativity and Gravitation, 33, 479 (2001); Erratum: 35, 939 (2003); astro-ph/0005084 v2].

[2] M.A.Ivanov, gr-qc/0009043; Proc. of the Int. Symp. "Frontiers of Fundamental Physics 4" (9-13 Dec 2000, Hyderabad, India), Eds B.G. Sidharth and M.V. Altaisky, Kluwer Academic/Plenum Publishers, August 2001; Proc. of the 4th Edoardo Amaldi Conference on Gravitational Waves (Perth, Western Australia, 8-13 July 2001) Class. Quantum Grav. 19, 1351 (2002).

[3] A.G. Riess et al. AJ 116, 1009 (1998).

[4] Anderson, J.D. et al. Phys. Rev. Lett., 1998, v.81, p. 2858; Phys. Rev. D65 (2002) 082004. gr-qc/0104064 v4]

[5] M.A.Ivanov. Model of graviton-dusty universe. gr-qc/0107047

[6] M.A.Ivanov. Screening the graviton background, graviton pairing, and Newtonian gravity. gr-qc/0207006 
[7] W. L. Freedman et al. ApJ, 553 (2001) 47.

[8] J.A. Willick, Puneet Batra. ApJ, 548 (2001) 564.

[9] S. Carlip. Quantum gravity: a progress report. gr-qc/0108040 